\title{
Research Paper: Reliability and Validity of Persian Version of Performance-oriented Mobility Assessment (POMA) in Community-dwelling Iranian Older Adults: Psychometric Properties
}

\author{
Bahman Moulodi $^{1}$ (D), Akram Azad ${ }^{1 *}$ (D), Ghorban Taghizadeh' ${ }^{1}$ (D), Mahtab Roohi-Azizi² ${ }^{\text {(D) }}$, Parvaneh Mohammadi ${ }^{1}$ (D)
}

1. Rehabilitation Research Center, Department of Occupational Therapy, School of Rehabilitation Sciences, Iran University of Medical Sciences, Tehran, Iran 2. Rehabilitation Research Center, Department of Basic Sciences, School of Rehabilitation Sciences, Iran University of Medical Science, Tehran, Iran

\begin{tabular}{|c|c|}
\hline $\begin{array}{l}\text { Use your device to scan and } \\
\text { read the article online }\end{array}$ & Chtation: Moulodi B, Azad A. Taghizadeh T, Roohi-Azizi M, Mohammadi P. Reliability and Validity of Persian Version of \\
\hline 回死神回 & Performance-oriented Mobility Assessment (POMA) in Community-dwelling Iranian Older Adults: Psychometric Properties. \\
\hline 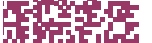 & Iranian Rehabilitation Journal. 2020; 18(1):39-48. http://dx.doi.org/10.32598/irj.18.1.626.5 \\
\hline 回etsin & doishttp://dx.doi.org/10.32598/irj.18.1.626.5 \\
\hline
\end{tabular}

\section{(i) (3)}

Article info:

Received: 11 Aug 2019

Accepted: 02 Nov 2019

Available Online: 01 Dec 2019

\section{Keywords:}

Elderly, Fall, Gait, Mobility,

Balance, Reliability, Validity

\section{ABSTRACT}

Objectives: An appropriate and accurate assessment tool is needed to predict the risk of falling in older adults. This study aimed at investigating the construct validity, factor analysis, internal consistency, test-retest and inter-rater reliability, and ceiling/floor effects of the Persian version of Performance-oriented Mobility Assessment (POMA) in community-dwelling elderly.

Methods: One hundred and forty-five older adults aged 65 years and older (mean age: 73.68 years) were recruited from daily care centers of Tehran by convenience sampling method. Timed Up and Go (TUG) test and Berg Balance Scale (BBS) were used to investigate the construct validity of the POMA. Test-retest (7-14 days with interval) and inter-rater reliability of the gait and balance subscales and the total score of the POMA were determined by Intraclass Correlation Coefficient $\left(\mathrm{ICC}_{2,1}\right)$.

Results: A moderate to very high correlation $(\mathrm{r}=0.67-0.9, \mathrm{P} \leq 0.05)$ was found between the tota score of the POMA and BBS, Fullerton Advanced Balance Scale, step test (right and left) TUG, Dynamic Gait index, and walking speed, whereas the correlation between the total score of the POMA and step length was poor $(\mathrm{r}=0.39, \mathrm{P} \leq 0.05)$. The results of confirmatory factor analysis showed a poor goodness-of-fit of POMA with the two-factor model (balance and gait) in community-dwelling elderly. Internal consistency (Cronbach's alpha=0.94), test-retest reliability $\left.\left(\mathrm{ICC}_{2,1}\right)=0.97\right)$, as well as inter-rater reliability $\left.\left(\mathrm{ICC}_{2,1}\right)=0.92\right)$ of the POMA were excellent. The results revealed no floor effect for the total score of the POMA; however, its ceiling effect was $3.44 \%$.

Discussion: The Persian version of POMA showed excellent psychometric properties for evaluating different aspects of balance in community-dwelling elderly.

\section{* Corresponding Author:}

Akram Azad, PhD.

Address: Rehabilitation Research Center, Department of Occupational Therapy, School of Rehabilitation Sciences, Iran University of Medical Sciences, Tehran, Iran.

Tel: +98 (21) 22228051

E-mail:azad.a@iums.ac.ir 


\section{Highlights}

- Internal consistency of the balance and gait subscores and all items of the Performance-Oriented Mobility Assessment was excellent.

- Test-retest and inter-rater reliability of the balance and gait subscores and the total score of the POMA was almost perfect agreement.

- The correlation between the total score of the POMA and the results of the BBS, FAB, DGI, walking speed, and step test were acceptable.

- Factor analysis of the POMA supported a two-factor model.

\section{Plain Language Summary}

Balance and gait impairments are two important risk factors for falling in older adults. Performance-oriented Mobility Assessment (POMA) is one of the functional measures used to evaluate balance, gait, and fall risk. Similar to other functional scales, evaluating psychometric properties of the POMA followed by the accurate translation and crosscultural adaptation for the target population and culture are needed to use this scale in different population groups. In this regard, this study was conducted to evaluate the psychometric properties of the Persian version of POMA in community-dwelling older adults and the results showed its excellent psychometric properties in this population.

\section{Introduction}

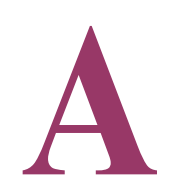

ccording to WHO (World Health Organization), aging is a biologic and dynamic process, which is uncontrollable by the human. A person aged over 65 years is considered as old [1]. The Elderly is one of the critical stages of life and the elderly population accounts for a high percentage of the global population in the near future. The world's elderly population is estimated to reach $25 \%$ by 2030 [2]. Several factors are involved in threatening the health, decreasing the quality of life and increasing the cost of care in the elderly. Falling is one of these factors, which is more likely to happen by aging, and about one-third of the elderly over 60 years experience falling during a year [3].

Balance impairments increase the falling risk. Falling may result in numerous physical (e.g., different types of fracture), psychological, social, and economic complications. Among psychological complications of falling, fear of falling is of particular importance, because it may restrict physical activities (due to decreased self-confidence and self-efficacy), increase the care by family or carers, and finally decrease the motor function as well as the independence of the elderly. As a result, fear of falling and its consequences in the elderly may impose huge costs on the health care systems [4].
Hence, researchers studying elderly and therapists have been looking to find appropriate and sensitive assessment tools to predict, estimate, and evaluate functional balance and mobility as well as falling and fear of falling in this population. Different tests/tools have been developed to achieve this goal, which have their own advantages [4]. Several tests have been developed for gait evaluation (e.g. Timed Up and Go test (TUG), Dynamic Gait Index (DGI)), as well as balance assessment (e.g. Berg Balance Scale (BBS), Fullerton Advanced Balance Scale (FAB), Step Test) in the elderly. However, Tinetti et al. developed Performance-Oriented Mobility Assessment (POMA) to evaluate both gait and balance in the elderly.

The POMA test has the best predictive validity for fall risk in the elderly compared with the TUG and FRT tests. POMA is easily administered and provides useful information on the ability of older people to assess mobility. The POMA test can be administered in less than 5 min and is more feasible than many other clinical trials, such as BBS [5]. This study was conducted to assess the possibility of using the Persian version of the POMA in Iranian studies [6].

Applicability, low cost for implementation as well as high reliability and validity are important advantages of the POMA [7]. Considering the growing population of the elderly in Iran, having an inexpensive assessment tool with appropriate psychometric properties is neces- 
sary for diagnosis and predicting balance and gait impairments in the elderly. Therefore, this study aimed at investigating the psychometric properties of the POMA in Iranian community-dwelling elderly.

\section{Methods}

The current research was a non-experimental cross-sectional study using a Persian version of the POMA. After obtaining permission to translate the POMA into Persian from the developer, the translation process was conducted according to the international quality of life assessment process. First, forward translation of the POMA was done by two native Persian translators from English to Persian language and two Persian versions were reviewed in a specialized panel resulting in an agreed Persian version of POMA. Then, backward translation was performed by two other translators and the final translation was adapted to the original version of the POMA and ultimately, the final Persian version of POMA was prepared. The research process lasted from May 2017 to May 2019.

Participants were recruited from daily care and rehabilitation centers of Tehran by convenience sampling method. The demographic characteristics of the partici- pants are presented in Table 1. Inclusion criteria were as follow: age $\geq 65$ years, having the ability to walk $6 \mathrm{~m}$ with or without assistive device and an acceptable level of cognitive function (i.e., score 18 or greater at minimental state examination) [8]. Subjects were excluded if they had neuro-musculoskeletal disorders (leading to inability to stand and walk) or dementia/ Alzheimer disease (leading to inability to understand the examiner's instructions), and a history of using lower extremity prosthesis [1]. All participants signed an informed consent form before data collection.

After completing a demographic questionnaire (through an interview or using medical record), an occupational therapy expert assessed participants by POMA, BBS, TUG, FAB, Step test (right and left), DGI, walking speed, and step length tests in two 45-minute sessions. Table 2 indicates the descriptive statistics of functional balance and mobility tests used in the current study. The tests were randomly carried out. Participants were allowed to rest whenever they felt tired. Inter-rater reliability of the Persian version of POMA was investigated by the cooperation of another experienced occupational therapist. An interval of seven or fourteen days was considered between test and retest [1].

Table 1. Demographic characteristics of the participants $(n=145)$

\begin{tabular}{|c|c|c|}
\hline \multicolumn{2}{|c|}{ Qualitative Variables } & \multirow{3}{*}{$\begin{array}{c}\text { Frequency (\%) } \\
80(55.17)\end{array}$} \\
\hline & Female & \\
\hline Gender & & \\
\hline & Male & $45(44.83)$ \\
\hline \multirow{2}{*}{ Life style } & Living alone & $17(11.7)$ \\
\hline & Living with family & $128(88.3)$ \\
\hline \multirow{2}{*}{ Fall history } & Yes & $46(31.7)$ \\
\hline & No & $99(68.3)$ \\
\hline \multirow[b]{2}{*}{ Using assisting devi } & Yes & $4(2.75)$ \\
\hline & No & $141(97.25)$ \\
\hline \multicolumn{2}{|c|}{ Quantitative Variables } & Mean \pm SD \\
\hline \multicolumn{2}{|c|}{ Age (y) } & $73.68 \pm 7.03$ \\
\hline \multicolumn{2}{|c|}{ Weight (kg) } & $76.13 \pm 11.68$ \\
\hline \multicolumn{2}{|c|}{ Height (cm) } & $165.73 \pm 9.58$ \\
\hline \multicolumn{2}{|c|}{$\mathrm{BMI}\left(\mathrm{m}^{2} / \mathrm{kg}\right)$} & $27.87 \pm 4.70$ \\
\hline \multicolumn{2}{|c|}{ Mini-mental state examination } & $26.65 \pm 2.95$ \\
\hline
\end{tabular}


Table 2. Mean $\pm S D$ of the functional balance and mobility tests in the elderly participated in this study $(n=145)$

\begin{tabular}{|c|c|}
\hline Test & Mean \pm SD \\
\hline Total score & $19.15 \pm 6.23$ \\
\hline Balance score & $11.48 \pm 3.77$ \\
\hline Gait score & $7.57 \pm 2.70$ \\
\hline BBS & $38.39 \pm 11.45$ \\
\hline FAB & $23.77 \pm 9.12$ \\
\hline Step Test (Right leg) (n/15s) & $5.10 \pm 2.40$ \\
\hline Step Test (Left leg) (n/15s) & $5.06 \pm 2.36$ \\
\hline Walking Speed (m/s) & $0.62 \pm 0.16$ \\
\hline Step Length $(\mathrm{cm})$ & $40.55 \pm 7.56$ \\
\hline TUG (s) & $11.60 \pm 0.44$ \\
\hline DGI & $15.13 \pm 5.73$ \\
\hline
\end{tabular}

Iranian Rehabilitation Journal

POMA: Performance Oriented Mobility Assessment; BBS: Berg Balance Scale; FAB: Fullerton Advanced Balance Scale;

TUG: Timed Up and Go; DGI: Dynamic Gait Index

\section{Instruments}

Performance-oriented Mobility Assessment (POMA): Tinneti developed the POMA in 1986 to evaluate fall risk in the elderly and made some changes in its items in $1993[1,8,9]$. This test measures both balance (9 items by a total score of $0-16$ ) and gait ( 7 items by a total score of $0-12$ ). A total score of the POMA ranges from 0 to 28 , which score $>24,19-24$, and $<19$ indicates low, medium and high fall risk, respectively [1, 8, 9]. It takes 5-10 minutes to complete the test. The advantage of the POMA over other balance tests is the possibility of assessing both balance and gait $[1,8,9]$. Moreover, the POMA is easily applicable $[1,8,9]$.

Berg Balance Scale (BBS): This scale, as a gold standard, was developed by Berg et al. in 1998 to assess functional balance, consisting of 14 items. Some equipment is needed to perform the test. Each item is scored from 0-4, leading to total score of 0-56. Impaired balance, and acceptable and good balance are characterized by a BBS score of 0-20, 21-40, and 41-56, respectively [1, 4, 6]. The reliability and validity of the Persian version of BBS have been established by Salavati et al. (2012) [10].

Timed Up and Go test (TUG): TUG is a measure of functional mobility developed by Richardson and Podsiadlo in 1991. The examiner records the time required to stand from a chair, walk 3 meters, turn around, and walk back to the chair by a stopwatch. The TUG time of 0-10s, 10-20s, and 20-30s indicates complete independence, marked dependence, and complete dependence of an individual in mobility, respectively. The TUG time of $13 \mathrm{~s}$ and greater is associated with increased fall risk in the elderly $[1,11]$. Kamrani et al. (2010) reported excellent reliability of the TUG in Iranian elderly [12].

Fullerton Advanced Balance Scale (FAB): This scale was developed by Rose et al. (2006) for evaluation of the balance in the elderly. It consists of 10 functional tasks assessing sensory, motor, and musculoskeletal systems involved in balance and mobility during both static and dynamic conditions. The examiner explains the subject of how to perform each task and the quality of performing each task is scored 0-4. A total FAB score of less than 25 indicates fall risk. The time required for completing the test is 10-12 min. The following equipment is needed to perform the FAB test: a stopwatch, pencil, $30 \mathrm{~cm}$ ruler, stool, masking tape, two foams and non-slippery pieces $[1,13]$.

Dynamic Gait Index (DGI): This index includes 8 gaitrelated items scoring from 0 (worst performance) to 3 (best performance). The maximum total score of the DGI is 24 and score 19 or less indicates fall risk. The DGI score of 22 or higher represents enough safety and no or least fall risk during gait. This test can be conduct- 
ed in 10-15 min with minimal equipment $[4,12]$. The reliability and validity of the DGI have been established by Abdiani et al. (2014) in Iranian elderly [2].

Step length: In this test, the subject is asked to walk 10 $\mathrm{m}$ and step length $(\mathrm{cm})$ is obtained thorough dividing the walking distance by the number of steps [14].

Walking speed: This test was developed by Sally in 1992 to assess functional mobility. Walking speed is determined by measuring the time required to walk a distance of 5 meters. The test environment should be completely unobstructed and well-marked at 0 and $5 \mathrm{~m}$. At the beginning of the test, the subject was placed behind the 0-meter start line asking to walk at his/her normal speed and not slow down a few steps after crossing the 5-meter line. This test is very sensitive, applicable to anyone who can walk $5 \mathrm{~m}$ and preferable to other tests that measure walking speed. The test is repeated 3 times and the average time is recorded as walking speed. Excellent reliability for this test has shown in the elderly population [15].

Step test: It is a measure of functional balance developed by Hill in 1996 and determines the number of times an individual can step on a stair in $15 \mathrm{~s}$. The subject should stand in front of a stair with a height of 15 $\mathrm{cm}$ while the distance between his feet is $10 \mathrm{~cm}$ and the distance between his thumb tip to the stair is $5 \mathrm{~cm}$. Following instruction is given to the subject: "When I say "start", put one of your feet up the stairs and put it back on the floor at maximum speed and do it again, until I say "stop". This process is repeated for the other foot. Good validity and reliability have been reported for this tes [4].

\section{Data analysis}

Normal distribution of data was assessed by the Kolmogorov-Smirnov test. To investigate construct validity, the correlation of balance and gait subscores, as well as the total score of the POMA with BBS, TUG, FAB, DGI, step length, walking speed and step test was determined using Pearson correlation coefficient. The correlation values of $\geq 0.9,0.68-0.89,0.36-0.67$, and $<0.35$ represent very strong, strong, moderate, and poor correlation, respectively [16]. Confirmatory Factor Analysis was done to further investigate the construct validity using a two-factor model, which its goodness-of-fit was evaluated by different indices, such as Goodness of Fit Index (GFI), Adjusted Goodness of Fit Index (AGFI), Normed Fit Index (NFI), Tucker-Levis Index (TLI), Comparative Fit Index (CFI), and Root Mean Square Error of Approximation (RMSEA). RMSEA values of less than $0.05,0.05-0.08,0.08-0.1$, and greater than 0.1 are considered as good, acceptable, marginal, and weak, respectively. A good fit is characterized by TLI and NFI values of $\geq 0.9$ [17-19].

Test-retest and inter-rater reliability of the balance and gait subscores and total score of the POMA was determined by Intraclass Correlation Coefficient $\left(\mathrm{ICC}_{2,1}\right)$, which its value of $0.6-0.8$, and greater than 0.8 indicates moderate and excellent reliability, respectively. Standard Error of Measurement (SEM) and Minimal Detectable Change (MDC) was calculated according to the following formulas: $\mathrm{SEM}=\mathrm{SD} \sqrt{1-\mathrm{ICC}}$ and $\mathrm{MDC}=\mathrm{SEM}$ $\sqrt{2} \times 1.96[8,20]$. Internal consistency was measured using Cronbach's alpha coefficient with a minimum acceptable value of 0.7 [16].

Floor/ceiling effects of total scores indicate how the scores accumulate at the bottom of the benchmark or ceiling [21]. The presence of floor and ceiling effect restricts the ability to discriminate evaluations and outcomes scores and thereby impede the functional usefulness of treatment during the follow-up period [22]. In addition, most clinical instruments have a floor and ceiling effect and, due to the high ceiling, effect in tests, such as BBS (Functional Balance) and DGI (Functional Mobility) in the community-dwelling older adults, the necessity of calculation and evaluation floor and ceiling effect appears necessary in the POMA test and in the community-dwelling older adults [23, 24]. Floor/ ceiling effects were determined by the percentage of the participants who obtained the minimum or maximum possible POMA score and considered acceptable if they were less than $15 \%$ of the sample. Statistical analysis was done using SPSS v.13 and the significance level was considered at $\mathrm{P} \leq 0.05$.

\section{Results}

One hundred and forty-five older adults aged 65 years and older by Mean \pm SD age of $73.68 \pm 7.03$ years, body mass index of $27.87 \pm 4.70$ and mini-mental state examination of $26.65 \pm 2.95$ participated in this study. The results of the Kolmogorov-Smirnov test showed normal distribution of data obtained by different tests $(\mathrm{P}>0.05)$.

\section{Validity}

The results revealed a poor to very strong direct correlation between the total score of POMA and the results of BBS, FAB, DGI, walking speed, step test (right and left legs) and step length $(\mathrm{r}=0.39-0.90)$, whereas there was a strong inverse correlation between it and TUG $(\mathrm{r}=-0.75)$. Balance and gait subscores of the POMA showed a poor 
Table 3. Correlation of the balance and gait subscores and total score of POMA with Step Length, Walking Speed, DGI, TUG, Step Test (right \& left), FAB, and BBS

\begin{tabular}{|c|c|c|c|c|c|c|c|c|c|c|c|c|c|c|c|c|}
\hline \multirow[t]{2}{*}{ Variables } & \multicolumn{2}{|c|}{ BBS } & \multicolumn{2}{|c|}{ FAB } & \multicolumn{2}{|c|}{$\begin{array}{c}\text { Step Test } \\
\text { Right } \\
\text { (n/15s) }\end{array}$} & \multicolumn{2}{|c|}{$\begin{array}{l}\text { Step Test } \\
\text { Left (n/15s) }\end{array}$} & \multicolumn{2}{|c|}{ TUG (s) } & \multicolumn{2}{|c|}{ DGI } & \multicolumn{2}{|c|}{$\begin{array}{c}\text { Walking } \\
\text { Speed } \\
(\mathrm{m} / \mathrm{s})\end{array}$} & \multicolumn{2}{|c|}{$\begin{array}{l}\text { Step } \\
\text { Length } \\
(\mathrm{cm})\end{array}$} \\
\hline & $r$ & $p$ & $r$ & p & $r$ & $\mathbf{P}$ & $r$ & p & $r$ & $\mathbf{P}$ & $r$ & $\mathbf{P}$ & $r$ & $\mathbf{P}$ & $r$ & $p$ \\
\hline $\begin{array}{l}\text { Balance } \\
\text { (sub scale) }\end{array}$ & 0.89 & 0.00 & 0.85 & 0.00 & 0.65 & 0.00 & 0.65 & 0.00 & -0.73 & 0.00 & 0.79 & 0.00 & 0.65 & 0.00 & 0.42 & 0.00 \\
\hline $\begin{array}{c}\text { Gait } \\
\text { (sub scale) }\end{array}$ & 0.85 & 0.00 & 0.77 & 0.00 & 0.67 & 0.00 & 0.65 & 0.00 & -0.73 & 0.00 & 0.78 & 0.00 & 0.63 & 0.00 & 0.34 & 0.00 \\
\hline POMA Total & 0.90 & 0.00 & 0.84 & 0.00 & 0.68 & 0.00 & 0.67 & 0.00 & -0.75 & 0.00 & 0.82 & 0.00 & 0.66 & 0.00 & 0.39 & 0.00 \\
\hline
\end{tabular}

Пranian Rehabilitation \ournal

POMA: Performance Oriented Mobility Assessment; BBS: Berg Balance Scale; FAB: Fullerton Advanced Balance Scale; TUG: Timed Up and Go; DGI: Dynamic Gait Index

to strong direct correlation with BBS, FAB, DGI, walking speed, step test (right and left legs) and step length $(\mathrm{r}=0.34-0.89)$, as well as a strong inverse association with TUG $(\mathrm{r}=-0.73)$ (Table 3).

\section{Factor Analysis}

RMSEA value of the current study (0.12) indicated poor fit, whereas GFI and AGFI values (0.76 and 0.68, respectively) showed a moderate fit and TLI and NFI values ( 0.8 and 0.83 , respectively) were less than the required value for good fit (i.e., $\geq 0.9$ ). Thus, POMA scores obtained in this study showed a poor fit with the twofactor model (Figure 1).

\section{Reliability}

The results showed excellent internal consistency of all items of POMA, as well as balance and gait subscales (POMA total: $\alpha=0.94$; balance subscale: $\alpha=0.91$; gait subscale: $\alpha=0.84$ ). Inter-item correlations ranged 0.27 to 0.76 . The correlation between each item and the total score of the POMA was 0.52-0.82. The range of Cronbach's alpha correlation after the deletion of each item was 0.93-0.94.

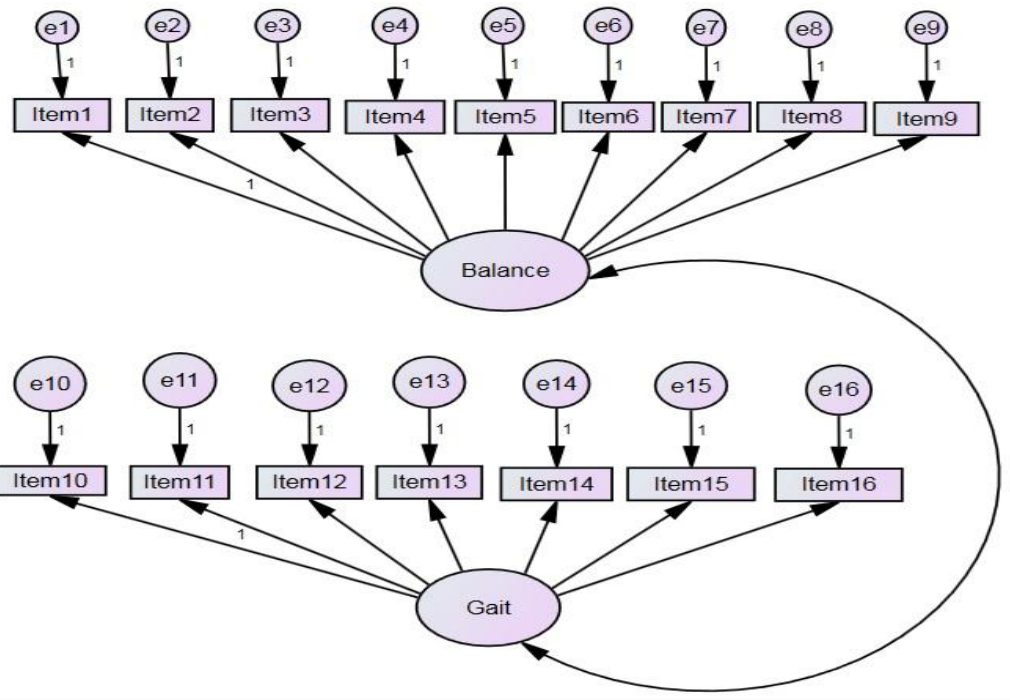

Iranian Rehabilitation Journal

Figure 1. Confirmatory factor analysis of the Persian version of POMA in Iranian community-dwelling elderly

(Model fit indices: $\mathrm{GFI}=0.76, \mathrm{AGFI}=0.68, \mathrm{NFI}=0.80, \mathrm{TLI}=0.83, \mathrm{CFI}=0.85, \mathrm{RMSEA}=0.12$ ).

GFI: Goodness-of-Fit Index; AGFI: Adjusted Goodness-of-Fit Index; NFI: Normed Fit Index; TLI: Tucker-Lewis Index;

CFI: Comparative Fit Index; RMSEA: Root Mean Square Error of Approximation 
Table 4. The results of relative and absolute test-retest and inter-rater reliability of the Persian version of POMA in Iranian community-dwelling elderly $(\mathrm{n}=145)$

\begin{tabular}{ccccccccc}
\hline \multirow{2}{*}{ POMA } & \multicolumn{3}{c}{ Test-retest } & \multicolumn{3}{c}{ Inter-tester } \\
\cline { 2 - 9 } & ICC (\%95 CI) & P & SEM & MDC & ICC (\%95CI) & P & SEM & MDC \\
\hline \multirow{2}{*}{ Balance } & $0.95(0.93-0.97)$ & 0.00 & 0.816 & 2.26 & $0.90(0.86-0.93)$ & 0.00 & 1.14 & 3.16 \\
Gait & $0.96(0.94-0.97)$ & 0.00 & 0.54 & 1.48 & $0.90(0.87-0.93)$ & 0.00 & 0.86 & 2.38 \\
Total & $0.97(0.96-0.98)$ & 0.00 & 1.05 & 2.91 & $0.92(0.89-0.94)$ & 0.00 & 1.72 & 4.76 \\
\hline
\end{tabular}

Iranian Rehabilitation Dourna

POMA: Performance Oriented Mobility Assessment; SEM: Standard Error of Measurement Minimal; MDC: Detectable Change

Table 5. Test-retest and inter-rater agreement (Kappa's coefficient) for each item of the Persian version of Performance-oriented Mobility Assessment (POMA) in Iranian community-dwelling elderly

\begin{tabular}{|c|c|c|c|c|c|c|c|}
\hline \multirow{2}{*}{\multicolumn{2}{|c|}{ POMA }} & \multicolumn{3}{|c|}{ Test-retest } & \multicolumn{3}{|c|}{ Inter-tester } \\
\hline & & kappa & $\mathbf{P}$ & Strength & kappa & p & Strength \\
\hline \multirow{9}{*}{$\begin{array}{l}\stackrel{\Xi}{ } \\
\frac{\pi}{\pi} \\
\frac{\infty}{0}\end{array}$} & 1 & - & - & - & - & - & - \\
\hline & 2 & 0.84 & 0.00 & Almost perfect agreement & 0.73 & 0.00 & Substantial agreement \\
\hline & 3 & 0.73 & 0.00 & Substantial agreement & 0.50 & 0.00 & Moderate agreement \\
\hline & 4 & 0.88 & 0.00 & Almost perfect agreement & 0.74 & 0.00 & Substantial agreement \\
\hline & 5 & 0.84 & 0.00 & Almost perfect agreement & 0.74 & 0.00 & Substantial agreement \\
\hline & 6 & 0.83 & 0.00 & Almost perfect agreement & 0.65 & 0.00 & Substantial agreement \\
\hline & 7 & 0.84 & 0.00 & Almost perfect agreement & 0.76 & 0.00 & Almost perfect agreement \\
\hline & 8 & 0.80 & 0.00 & Almost perfect agreement & 0.66 & 0.00 & Substantial agreement \\
\hline & 9 & 0.84 & 0.00 & Almost perfect agreement & 0.74 & 0.00 & Substantial agreement \\
\hline \multirow{7}{*}{ 若 } & 10 & 0.85 & 0.00 & Almost perfect agreement & 0.75 & 0.00 & Substantial agreement \\
\hline & 11 & 0.88 & 0.00 & Almost perfect agreement & 0.71 & 0.00 & Substantial agreement \\
\hline & 12 & 0.90 & 0.00 & Almost perfect agreement & 0.75 & 0.00 & Substantial agreement \\
\hline & 13 & -0.12 & 0.00 & Poor agreement & 0.70 & 0.00 & Substantial agreement \\
\hline & 14 & 0.84 & 0.00 & Almost perfect agreement & 0.71 & 0.00 & Substantial agreement \\
\hline & 15 & 0.84 & 0.00 & Almost perfect agreement & 0.70 & 0.00 & Substantial agreement \\
\hline & 16 & 0.93 & 0.00 & Almost perfect agreement & 0.83 & 0.00 & Almost perfect \\
\hline
\end{tabular}

Relative test-retest reliability (ICC) of balance and gait subscores and total score of the POMA were 0.95, 0.96, and 0.97 , respectively. Absolute test-retest reliability (SEM) of balance and gait subscores and total score of the POMA were $0.82,0.54$, and 1.05 , respectively. The MDC of these scores was 2.26, 1.48, and 2.91, respectively. Relative inter-rater reliability of balance and gait subscores and total score of the POMA were 0.90, 0.90, and 0.92 , respectively. In addition, absolute test-retest reliability of these scores was $1.14,0.86$, and 1.72 , respectively, and their MDC values were 3.16, 2.38, and 4.76, respectively (Table 4).
The Kappa's coefficient for test-retest and inter-rater evaluation of each item of POMA ranged 0.12-0.93 and $0.50-0.83$, indicating poor to almost perfect and moderate to the almost perfect agreement, respectively (Table 5).

The results of this study showed no floor effect for none of the subscores or total score of the POMA. The ceiling effect for balance and gait subscores and total score of the POMA were $30.34 \%, 4.82 \%$, and $3.44 \%$, respectively. 


\section{Discussion}

The aim of this study was to investigate the construct validity, factor analysis, and test-retest and inter-rater reliability of the Persian version of POMA in Iranian community-dwelling elderly.

The results showed a strong to the very strong correlation between the POMA and BBS, FAB, and DGI. One possible reason for this finding may be similar tasks in POMA and BBS (e.g., balance in sitting and standing conditions, standing with open and closed eyes, and turning 360 degrees), and FAB (e.g., standing with open and closed eyes and turning 360 degrees). The strong correlation between POMA and DGI also can be explained by the fact that almost half of POMA items evaluate different aspects of gait. These results are in agreement with those reported by Yücel et al. (using the Turkish version of POMA), Cho et al., and Faber et al. who revealed a moderate to very strong correlation between total score of the POMA and BBS in the elderly $[1,8,20,25]$.

Further, the results revealed a strong inverse correlation between the total score of POMA and TUG. The current study also found a poor to moderate correlation of total score of POMA with walking speed, step test, and step length. The results also indicated that balance and gait subscores of the POMA were poorly to moderately correlated with BBS, DGI, FAB, walking speed, step test and step length in a direct manner, whereas they showed a strong inverse correlation with TUG. The poor correlation between POMA and step length observed in this study may be due to no similarity among POMA items and step length test [26].

In factor analysis, RMSEA value indicated poor fit and GFI and AGFI values showed moderate fit. TLI and NFI values were 0.8 and 0.83 , respectively. Therefore, the twofactor model has a poor fit with the data obtained in the current study. Hence, investigating more models by deleting the items may be helpful to improve the overall goodness-of-fit of the model. However, the aim of the present study was to investigate a literature-based two-factor model, which was not modulating. Therefore, this issue should be investigated in more detail in future studies.

Internal consistency measures different items of a scale that propose to measure the same general construct produce similar scores [5]. An excellent internal consistency was found for all items and balance and gait subscales of the POMA in this study. This finding was in line with that of Yücel et al. who used the Turkish version of POMA in the elderly [1]. The results of the current study indicated excellent test-retest reliability of POMA $(\mathrm{ICC}=0.97)$ for assessing Iranian community-dwelling elderly. Previous studies also have reported excellent test-retest reliability of the POMA in the elderly and patients with Parkinson disease and multiple sclerosis $[1,8,11]$.

Consistent with Yücel et al. in Turkish elderly [1], we found excellent inter-rater reliability of POMA (ICC $=0.92$ ) in Iranian community-dwelling elderly. Except for the item 13, the test-retest and inter-rater agreement of the POMA was moderate to almost perfect, which was consistent with Kloos et al. study in patients with multiple sclerosis [11].

MDC, which indicated the minimum change that can be considered as a real change [27], was 2.91 and 4.76 in test-retest and inter-rater reliability, respectively. These MDC values are useful for therapists who study on the balance in the elderly because POMA scores higher than these values indicate real improvement of balance in the elderly following interventions.

The floor and ceiling effect of a scale indicates the accumulation of scores at the lower (floor) or upper (ceiling) end of the scale [5]. Floor effect was not observed in this study, however, the ceiling effect for balance and gait subscores and total score of the POMA were $30.34 \%, 4.82 \%$, and $3.44 \%$, respectively. This result was in accordance with the result of Faber et al. study in the elderly [8].

\section{Limitation}

We used a convenience sampling method, which should be considered as a potential limitation of this study. It is suggested to use the probability sampling method in future studies.

\section{Conclusion}

The results of this study showed that POMA has an acceptable test-retest and inter-rater reliability, as well as internal consistency for assessing balance and gait in the elderly.

\section{Ethical Considerations}

\section{Compliance with ethical guidelines}

This study was approved by the Ethics Committee of Iran University of Medical Sciences (Code: IR.IUMS. REC.1395.9413355003). 
Funding

This study was financially supported by Iran University of Medical Sciences.

\section{Authors' contributions}

Conceptualization, Supervision: Akram Azad; Methodology: Akram Azad, Ghorban Taghizadeh; Literature search: Bahman Moulodi, Mahtab Roohi-Azizi; Data collation: Bahman Moulodi, Parvaneh Mohammadi; Data entry and analysis: Bahman Moulodi, Ghorban Taghizadeh; Writing - original draft: Akram Azad, Ghorban Taghizadeh, Bahman Moulodi; Writing-review \& editing: Akram Azad, Ghorban Taghizadeh.

\section{Conflict of interest}

The authors declared no conflict of interests.

\section{Acknowledgments}

We would like to appreciate all participants and their families for their cooperation.

\section{References}

[1] Yücel SD, Sahin F, Dogu B, Sahin T, Kura B, Gursakal S. Reliability and validity of the Turkish version of the PerformanceOriented Mobility Assessment I. European Review of Aging and Physical Activity. 2012; 9(2):149-59. [DOI:10.1007/s11556012-0096-2

[2] Abdiani M, Golpayegani M, Khajavi D. [Validity and reliability of Persian version of Dynamic Gait Index in older men (Persian)]. Salmand: Iranian Journal of Ageing. 2014; 9(2):124-33.

[3] Salehi L, Shokravash B, Jamshidi E, Montazeri A. Physical activity in Iranian older adults who experienced fall during the past 12 months. BMC Geriatric. 2014; 14:115. [DOI:10.1186/1471-2318-14-115] [PMID] [PMCID]

[4] Raji P. [Functional balance tests (Persian)]. Audiotory and Vestibular Research. 2012; 21(4):1-9.

[5] Kegelmeyer DA, Kloos AD, Thomas KM, Kostyk SK. Reliability and validity of the Tinetti Mobility Test for individuals with Parkinson disease. Physical Therapy. 2007; 87(10):136978. [DOI:10.2522/ptj.20070007] [PMID]

[6] Azad A, Taghizadeh Gh, Khaneghini A. Assessment of the reliability of the Iranian version of the Berg Balance Scale in patients with Multiple Sclerosis. Acta Neurologica Taiwanica. 2011; 20(1):22-8

[7] Sterke CS, Huisman SL, Van Beeck EF, Looman CW, Van der Cammen Tj. Is the Tinetti Performance Oriented Mobility Assessment (POMA) a feasible and valid predictor of shortterm fall risk in nursing home residents with dementia? In- ternational Psychogeriatric. 2010; 22(2):254-63. [DOI:10.1017/ S1041610209991347] [PMID]

[8] Faber MJ, Bosscher RJ, van Wieringen PC. Clinimetric properties of the Performance-Oriented Mobility Assessment. Physical Therapy. 2006; 86(7):944-54. [DOI:10.1093/ ptj/86.7.944] [PMID]

[9] Tinetti ME. Performance-Oriented Assessment of Mobility problems in elderly patients. Journal of the American Geriatrics Society. 1986; 34(2):119-26. [DOI:10.1111/j.1532-5415.1986 tb05480.x] [PMID]

[10] Salavati M, Negahban H, Mazaheri M, Soleimanifar M, Hadadi M, Sefiddahti L, et al. The Persian version of the Berg Balance Scale: Inter and intra-rater reliability and construct validity in elderly adults. Disability and Rehabilitation. 2012; 34(20):1695-8. [DOI:10.3109/09638288.2012.660604] [PMID]

[11] Kloos A, Dal Bello-Haas V, Thome R. Interrater and intrarater reliability of the Tinetti Balance Test for individuals with amyotrophic lateral sclerosis. Journal of Neurologic Physical Therapy. 2004; 28(1):12-19. [DOI:10.1097/01. NPT.0000284773.87060.c8

[12] Akbari-Kamrani AA, Zamani-Sani S-H, Fathi-Rezai Z, Aghdasi MT. Concurrent validity of functional gait assessment, timed up and go, and gait speed tests in the Persian community-dwelling elderly. Iranian Rehabilitation Journal. 2010; 8(12):15-20

[13] Rose DJ, Lucchese N, Wiersma LD. Development of a multidimensional balance scale for use with functionally independent older adults. Archives of Physical Medicine and Rehabilitation. 2006; 87(11):1478-85. [DOI:10.1016/j. apmr.2006.07.263] [PMID]

[14] Eskandarnejad M, Taghizadeh S. [A comparison of distance perception in active and inactive elderly with an emphasis on physical activity (Persian)]. Iranian Journal of Geriatric Nursing. 2015; 2(1):79-90. [DOI:10.21859/jgn.2.3.81]

[15] Denise P, Stacy F, Debra K. Assessing the reliability and validity of a shorter walk test compared with the 10-meter walk test for measurements of gait speed in healthy, older adults. Journal of Geriatric Physical Therapy. 2013; 36(1):2430. [DOI:10.1519/JPT.0b013e318248e20d] [PMID]

[16] Bentler PM. Comparative fit indexes in structural models. Psychological Bulletin. 1990; 107(2):238-46 [DOI:10.1037/0033-2909.107.2.238] [PMID]

[17] Fabrigar LR, Wegener DT, MacCallum RC, Strahan EJ. Evaluating the use of exploratory factor analysis in psychological research. Psychological Methods. 1999; 4(3):272-99. [DOI:10.1037/1082-989X.4.3.272]

[18] Mulaik SA, James LR, Van Alstin J, Bennett N, Lind Sh, Stilwell CD. Evaluation of goodness-of-fit indices for structural equation models. Psychological Bulletin. 1989; 105(3):430 45. [DOI:10.1037/0033-2909.105.3.430]

[19] Fritz SL, Blanton S, Uswatte G, Taub E, Wolf SL. Minimal detectable change scores for the Wolf Motor Function Test Neurorehabilitation and Neural Repair. 2009; 23(7):662-7. [DOI:10.1177/1545968309335975] [PMID]

[20] Cho BL, Scarpace D, Alexander NB. Tests of stepping as indicators of mobility, balance, and fall risk in balance-impaired older adults. Journal of the American Geriatrics Society. 2004 52(7):1168-73. [DOI:10.1111/j.1532-5415.2004.52317.x] [PMID] 
[21] Naghdi S, Nakhostin-Ansari N, Raji P, Shamili A, Amini $\mathrm{M}$, Hasson S. Cross-cultural validation of the Persian version of the Functional Independence Measure for patients with stroke. Disability and Rehabilitation. 2016; 38(3):289-98. [DO I:10.3109/09638288.2015.1036173] [PMID]

[22] Boulez J, Canovas F, Duflos C, Dagneaux L. Are modern knee outcomes scores appropriate for evaluating patellofemoral degeneration in osteoarthritis? Evaluation of the ceiling and floor effect in knee outcomes scores. Orthopaedics \& Traumatology: Surgery \& Research. 2019; 105:599-603. [DOI:10.1016/j.otsr.2019.01.018] [PMID]

[23] Balasubramanian, CK. The community balance and mobility scale alleviates the ceiling effects observed in the currently used gait and balance assessments for the community-dwelling older adults. Journal of Geriatric Physical Therapy. 2015; 38(2):78-89. [DOI:10.1519/JPT.0000000000000024] [PMID]

[24] Ban B, Sevsek F, Rugelj D. A comparison of the ceiling effect between Berg Balance Scale and Mini-BESTest in a group of balance trained community-dwelling older adults. Physiotherapy Quarterly (formerly Fizjoterapia). 2017; 25(2):3-9. [DOI:10.5114/pq.2018.73368]

[25] Schoon Y, Weerdesteyn V, Stunnenberg A, Old Rikkert MG Sense and simplicity: Maximum step length is also reliable, feasible, and valid in very old adults. Journal of the American Geriatrics Society. 2010; 58(12):2444-5. [DOI:10.1111/j.15325415.2010.03193.x] [PMID]

[26] Ng SSM, Tse MMY, Kwong PWH, Fong ICK, Chan SH, Cheung TCH, et al. Reliability of the Maximal Step Length Test and its correlation with motor function in chronic stroke survivors. BioMed Research International. 2018 [DOI:10.1155/2018/6985963] [PMID] [PMCID]

[27] Donoghue D, Stokes EK. How much change is true change? The minimum detectable change of the Berg Balance Scale in elderly people. Journal of Rehabilitation Medicine. 2009; 41(5):343-6. [DOI:10.2340/16501977-0337] [PMID] 